\title{
«Analyse spatiale de l'information géographique»
}

\author{
Q. H. Dao \\ Département de Géographie, Université de Genève, Switzerland \\ Correspondence to: Q. H. Dao (hy.dao@unige.ch)
}

Caloz, R. and Collet, C.: Analyse spatiale de l'information géographique, Lausanne, Presses polytechniques et universitaires romandes, 383 pp., ISBN-13: 9782880749026, $€ 51.40,2011$.

Après «Systèmes d'information géographique en mode image» (1992) et «Précis de télédétection, Vol. 3: Traitements numériques d'images de télédétection» (2001) Régis Caloz (Ecole Polytechnique Fédérale de Lausanne) et Claude Collet (Université de Friboug) publient un nouveau livre en langue française dans le domaine de la géomatique. Cet ouvrage s'adresse à un public certes spécialisé mais que les auteurs souhaitent large: étudiants, enseignants, ingénieurs. Ses objectifs annoncés sont de permettre aux lecteurs de comprendre les fondements de l'analyse spatiale, de déterminer ses potentiels d'application, de mettre en ouvre des opérations de traitement et d'exercer un œil critique sur les résultats.

«L'analyse spatiale s'inscrit principalement dans deux phases du cycle de vie de l'information, lors de la modélisation de l'espace et lors de l'exploitation des informations» (p. 1). Cet extrait du début de l'ouvrage exprime la vision qu'ont les auteurs d'une analyse spatiale inscrite dans un processus itératif de transformation de l'information et résume la structure de l'ouvrage.

Le livre est en effet découpé en dix chapitres organisé en deux parties principales - modélisation de l'espace géographique (chapitres 1 à 4 ) et exploitation des informations (chapitres 6 à 9) - séparées par un chapitre thématique. Le chapitre 1 rappelle l'importance de la notion de «cycle de vie de l'information géographique» et des SIG comme des modèles évolutifs de la réalité. Le chapitre 2 présente les bases de la modélisation spatiale. Les chapitres 3 et 4 proposent une réflexion sur la nature des phénomènes modélisés (discontinus ou continus). Le chapitre 5 propose une entrée thématique sur le relief, avant quatre chapitres consacrés à des méthodes d'analyse spatiale. Le chapitre 6 traite des requêtes spatiales, c'est-à-dire des méthodes d'interrogation des bases de données géographiques. Les chapitres 7 et 8 abordent les calculs d'accessibilité et l'analyse multicritère. Le chapitre 9 est consacré à l' analyse des évolutions spatio-temporelles. Le chapitre 10 conclut le livre avec la question des incertitudes et de leur propagation.

Chaque chapitre propose trois niveaux d'approche: une introduction, une réflexion plus approfondie sur les phénomènes et traitements en jeu, une présentation d'aspects opérationnels, avant de se conclure par quelques suggestions de lecture et des «questions de révision». Une bibliographie générale est également fournie à la fin du livre.

Structuré par les deux idées fortes de «cycle de vie de l'information» et de «modèle de la réalité», le livre aborde un grand nombre de sujets de manière structurée et claire, notamment grâce à une illustration graphique abondante et élégante. Chaque chapitre peut se lire de manière indépendante et constitue un excellent point d'entrée vers le sujet concerné, tout en en proposant les bases essentielles (voir par exemple l'analyse factorielle dans le chapitre 8).

La présentation et les intitulés de certains chapitres peuvent parfois interpeller le lecteur. Ainsi, la question de l'accessibilité ne s'applique-t-elle qu'aux objets linéaires (chapitre 7) et les méthodes uni- et multivariées qu'aux objets zonaux (chapitre 8)?

Le chapitre conclusif rappelle de manière très opportune que l'incertitude - définie comme «l'intervalle à l'intérieur duquel la valeur est acceptée comme représentative du phénomène» - est consubstantielle à la notion d'information et que l'analyse spatiale procède par approximation de la réalité. Une approche scientifique implique une évaluation de la fiabilité de l'information produite, même si cela est souvent rendu difficile par l'hétérogénéité des données combinées pendant les traitements.

On aurait pu souhaiter voir cette conclusion, par ailleurs très pertinente, s'ouvrir vers d'autres méthodes d'analyse de l'information comme par exemple la géo-visualisation. Mais ceci n'enlève rien à la richesse et aux qualités pédagogiques de cet ouvrage qui devrait rapidement devenir un texte de référence pour le scientifique de l'information géographique. 